\title{
Index
}

Page numbers in italic indicate figures

Page numbers in bold indicate Operative Techniques

Abdominal examination, preoperative assessment 49

Acetabular plates 80

Achilles tendon 21

Acrylic resin ESF constructs 85

Actinomyces spp. 378

Adhesions 32

Adrenaline 18

Advanced locking plate system (ALPS) 82

AISI (American Iron and Steel Institute) grades of stainless steel 65

Alendronate 115

Allografts $121-2$

cancellous bone chips 121, 122

cortical grafts 121,122

demineralized bone matrix (DBM) 121-2

Alloplastic grafts 123-4

AMI see Area moment of inertia

Amoxicillin/clavulanate (co-amoxiclav) 59, 107, 382

Amputation

for quadriceps contracture 366

in bone neoplasia 114

open fractures 110

Anaesthesia 60

history of 1,2

Analgesia see Pain management

Aneurysmal bone cysts 117

Angiogenesis in fracture sites 33-4, 388

Angle-stable plates 5

Anisotropic materials 20

Antibiotic resistance 107

effects of biofilms $374-5$

plasmid-mediated transmission 375

Antibiotics 5

coating of implants 383

perioperative use 59

prophylactic use in open fractures 107

use in osteomyelitis $379,382-3,384-5$

Antisepsis, development of 1,2

$\mathrm{AO}$ (Arbeitgemeinschaft für Osteosynthesefragen) group 3, 363 new implants and instruments 5

principles for fracture management $5,6,55,56-7,72$

AOVET group 363

Apical ectodermal ridge 14, 15

Apophyseal fracture 8

Appendicular skeleton 12

Area moment of inertia (AMI) 21, 22, 25

of an implant 370-1

of K-wire-bone constructs 29

of orthopaedic wire 74

of plate and screw constructs $27,28-9$

Arthrodesis 60, 68, 393, 396

bone grafting 120

for carpal fractures 321

for quadriceps contracture 366

open fractures 110, 111

shoulder joint 198

stress riser effect 118
Arthrodesis plates 80

Arthroplasty 393

Articular fractures $8,9,40$

complete articular fracture 9

imaging 41

in juvenile animals 61,63

malunions 396

management of 60,63

non-unions 393

partial articular fracture 9

risk of osteoarthritis 55,60

Asepsis in fracture surgery 57-8

Aspergillus spp. 385

Atrophic non-union 389, 391

Autogenous grafts 120-1, 125

advantages 120

disadvantages 120

harvesting autogenous cancellous bone 125

types of 120-1

Avulsion fracture 8

in juvenile animals 62

radiographic evidence of 40

Axial skeleton 12

Bacterial biofilms 73, 374-5

Bacterial osteomyelitis 112, 113

Bacteroides spp. 378

Bandaging of fractures 59, 63

Basal fracture 8

Basic surgical techniques 88-105

Operative Techniques 91-105

patient set-up and positioning 88, 90

safety checklists 88,89

surgical planning 88,89

theatre set-up and organization 88

trolley set-up 90

see also Fracture surgery and specific fractures

Bench-mounted press 69,70

Bending forces on bones and implants 21, 22

Bending irons/pliers 69

Beta-lactam antibiotics 59

Bethanechol 174

Bi-gnathic encircling and retaining device (BEARD) 152, 153, 161,162

Bioactive glass bone grafts 124

Biodegradable plates and screws 82

Biofilms 73, 374-5

Biological functions of bone 12

Biological healing, approach to fracture management 5-6, 109

Biological osteosynthesis 394 approach to fracture surgery 56-7 principles 126

Biologically active non-union of fractures see Viable non-union of fractures

Biologically inactive non-union of fractures see Non-viable non-union of fractures

Bisphosphonates 114, 115

Blastomyces dermatitidis 384 
Blood supply

factors affecting fracture site 388

influence on fracture healing 388

Bone

apparent shortening of 40

as an anisotropic material 20-1

brittle property 22,23

dry weight of mature bone 13

ductility of 22,23

embryology 13

material properties 21

stress-strain curve 21

Bone biopsy 114, 115

Bone cysts 117-18

Bone density, disuse osteopenia 46

Bone grafts $68,120-5$

allografts 121-2

alloplastic grafts 123-4

autogenous grafts 120-1, 125

bioactive glass 124

ceramics 123

composite alloplastic grafts 124

definitions 120

glass ionomer cements 124

growth factors 122-3

harvesting autogenous cancellous bone graft 125

ideal bone graft properties 124

in osteomyelitis $381-2$

indications for bone grafting 120

injectable calcium phosphate 123

mechanisms of enhancement of healing 120

mesenchymal stem cells 123

non-biological osteoconductive substrates 124

open fractures 109

tissue engineering 123

Type 1 collagen 124

Bone growth

capillary formation 13

endochondral ossification 13-14

intramembranous ossification 13, 14

mineralization 13

Bone healing (regeneration) 32-6

direct bone healing 32-3

indirect bone healing 33-5

mechanics of 23

see also Fracture healing

Bone-implant constructs

forces acting on 22

mechanics of 21,22

tension and compression surfaces of bones 22

Bone mechanobiology 16-17

Bone morphogenic proteins (BMPs) 13, 34, 121, 122-3, 388

Bone plates 77-82

ability to resist disruptive forces 72

biodegradable plates and screws 82

dynamic compression plates (DCPs) 78-9

functions of 77-8

locking plates $80-2$

maxillofacial fractures 154,155

modes of use 77-8

non-locking plates $78-80$

stress riser effect 118

types of 78-82

see also Plate and screw fixation

Bone remodelling 16

calcium regulatory pathways 18-19

effects of decreased mechanical load 16

environmental influences 17, 18

hormonal influences 17-18

non-mechanical influences 17-19

response to mechanical stress 16-17

Bone resorption 16

Bone screws 76-7

ability to resist disruptive forces 72

cancellous screws $76-7$

choice of type and size 21

cortical screws 76-7

head shearing off $368-9$

lag screw technique 77

morphology 76

non-self-tapping screws 77

plate screws 77 positional screws 77

pull-out strength 27,29

self-tapping screws 77

sizing 76,77

staged selective screw removal $35-6$

thread profiles 27

types for plate and screw fixation 27

Bone structure see Bone remodelling

Bone taps 69

Bone tumours see Neoplasia

Bone union, definition 386

Bones

biological functions 12

classification by shape 12-13

forces acting on 20-2

mechanical functions 12

skeletal anatomy 12-13

vasculature 55

Boxers, radial carpal bone fractures 60,321

Bridging fixation 56

Bridging plates 28-9, 104

Buprenorphine 60

Butterfly fragment fracture $8,23,23$

Calcaneal fractures 324-7

avulsion of the tuber calcis 324, 325

fracture/separation of the proximal calcaneal physis 324,325

fractures of the base 327,328

mid-body fractures 324-5, 325-6

slab fractures 327,327

Calcaneus 21

Calcinosis cutis 41

Calcitonin 19

Calcitriol 116

Calcium deposition as bone develops 13

Calcium levels in serum 115-16

Calcium phosphate injectable bone graft 123

Calcium regulatory pathways, influence on bone 18-19

Callus formation $32,34,35$ excessive 47

Calvaria fractures $149,159-60$

Canaliculi within bone 13

Cancellous bone chip allografts 121, 122

Cancellous screws 76-7

Capital fracture 8

Carbenicillin 382

Carbon fibre ESF connecting bars 85

Carpal arthrodesis plates 80

Carpal bones, anatomy of the carpus 319-20

Carpal contracture 363

Carpal fractures

accessory carpal bone 9,323-4

anatomy of the carpus 319-20

diagnosis 319

distal carpal bones 322

incidence of 319

nature of 319

preoperative planning 319

radial carpal bone $320-2$

comminuted or Y-shaped fractures 321

dorsal slab or chip fractures 320

fracture-luxation 322

oblique mid-body (parasagittal) fractures 320-1

palmar process 321-2

shearing injuries of the carpus 324

ulnar carpal bone 322

Carpal Operative Techniques

accessory carpal bone type I and II fractures, screw fixation 333-4

dorsal approach to the carpus 331

palmaromedial approach to the radial carpal bone 332

Carpus joint fractures, imaging 41

Cartilage formation 34

Cartilage precursor of bone 13-14

Casts for fractures $63,142-8$

avoiding fracture disease 366-7

cast application 143-5

cast biomechanics 142

cast construction 143-4

cast loosening 147

cast maintenance 145 
cast removal 145

complications of casts 146-7

costs 143,148

delayed union 147

effects of immobilization 146

effects on young dogs 146

fracture configuration 142

fracture location 142-3

fracture reduction 143

indications for 142-3

intended role of the patient 143

limb swelling caused by 146

malunion 147

non-union 147

pressure sores and infection 146, 147

properties of casting materials 143

re-fracture 147

risk of fracture disease $364-5$

signalment 143

use in juvenile animals 62

Cbfa1 (Runx2) transcription factor 13, 14

Cefalexin 382

Cefazolin 382

Ceftazidime 382

Cefuroxime 59

Cell signalling, role in fracture healing process 388

Centre of rotation of angulation (CORA) 395, 396

Cephalosporin antibiotics 59, 107, 382

Ceramic bone grafts 123

Cerclage tensioning devices 70

Cerclage wiring of fractures $3,29-30,72,73-4,96-7$

Chemotherapy 114

Chip fractures 8,60

Chisels 68-9

Chondrocytes 13, 14, 17, 34

Chondroitin sulphate 13

Chrondrosarcoma 112, 114

Circular external skeletal fixation (CESF) 3, 27, 86

Clindamycin 382

Clinical union of fractures, definition 386

Closed (simple) fracture 7

Clostridium spp. 376, 378

Cocaine- and amphetamine-regulated transcript (CART) gene 18

Coccidioides immitis 384

Codeine 60

COL1 gene mutations 117

Collagen fibres in bone $20-1$

Collagen-proteoglycan matrix (osteoid) 13

Collagen types I, II and III 34

Collagens, cartilage-specific 14

Colles' fracture 7

Comminuted fractures $7,8,23,32$

approach to fracture surgery 56-7

biological approach to treatment 5-6

complications 5

decision to reduce or not 24

Compartment syndrome 50

Complete articular fracture 9

Complete fracture 8

Complicated fracture 7

Complications of fracture healing 386-97

critical defect $388,389,391$

defective bone healing 386-8

definitions 386

delayed union 386, 393-4

factors affecting blood supply 388

failure of cell signalling mechanisms 388

fracture gap too large to heal $388,389,391$

fracture site biology 387-8

fracture site mechanics 387

incomplete extracellular matrix 388

infection 391, 392-3, 394

influence of interfragmentary strain (IFS) 387

influence of stress protection 387

malunion 386, 394-6

non-union 386, 388-93

radiographic evidence $44-7$

role of mesenchymal stem cells (MSCs) 386-7

Compound fractures 7

Compression, radiographic evidence of 40

Compression fractures 8,32

Compression plates 27-8
Computed tomography (CT) 10, 37, 41, 42

three-dimensional imaging of fractures 50-1

Computer analysis of fracture data 10

Condylar fracture 8

Congenital disorders affecting bone 18, 115

Congenital hyperparathyroidism 115

Congenital hypothyroidism 18

Connecting bars for ESF 85-6

Connecting clamps for ESF 84-5

Contact healing of fractures 32,33

Corrective osteotomy, surgical planning 395-6

Corrosion degradation of implants $372-3$

Cortical allografts 121, 122

Cortical bone, behaviour under different loadings 23

Cortical screws 76-7

Corticosteroids 171

Critical defect, adverse effects on bone healing 388, 389, 391

Cruciate ligament injury and disease 356

Cryptococcus neoformans 384

Curette 68, 69

Cutting cone of osteoclasts 16, 33

Cyclic fatigue failure of implants $4,5,45-6$

Cyclic micromovement 35

Cytokines $33,121,388$

Dachshunds, genetic test for collagen disorders 117

Debridement and drainage in osteomyelitis 379-80

Defect non-union of fractures 388, 389, 391

Delayed union of fractures $44,45,386,393-4$

Demineralized bone matrix (DBM) allografts 121-2

Dentinogenesis imperfecta 117

Dentoalveolar injuries 149

Depressed fracture 8

Dexamethasone 171

Diagnostic imaging see Imaging of fractures

Diaphyseal fracture 8

Diaphysis 12

Diazepam 171

Direct healing of fractures 32-3, 56

Discospondylitis 384-5

Distal fracture 8

Distal limb

anatomy 342

metacarpal bones 342

metatarsal bones 342

sesamoid bones 342

Distal limb fractures 41

amputation of the digits 347-8

combined ESF and temporary IM pinning 345

combined IM pinning and ESF 345

external skeletal fixation (ESF) 344

fractures of the digits $347-8$

IM techniques 344

in racing Greyhounds 342, 343, 345-6

metacarpal/metatarsal bones 342-6

metacarpal/metatarsal stress fractures 10

phalangeal fractures 347-8

physeal fractures 345

plate and screw fixation 343-4

sesamoid bones $346-7$

sesamoidectomy 347

surgical considerations 342

treatment by external coaptation 342

Distal limb Operative Techniques distal digital amputation $\mathbf{3 5 2}$

metacarpal/metatarsal fractures, IM pinning 348-9

permanent nail removal $\mathbf{3 5 1}$

Distraction osteogenesis 3

Disuse osteopenia 46

Dobermanns, polyostotic bone cysts 117

Dowel pinning of fractures 4

Draping for fracture surgery 58,59

Drill guides 69

Drills for orthopaedic surgery 66-7, 68

Dynamic compression plates (DCPs) 5, 27-9, 78-9, 102

Dynamization 35-6

Dystrophic non-union of fractures 389, 390-1

ECG, preoperative monitoring 49

Elastic deformation 20

Electromagnetic field therapy 393 
Ellis pins 25

Embryology of bone 13

Endochondral ossification 13-14, 34

Endocrine influences on bone 17-18

Endosteal callus 34, 35

Endosteal remodelling 16

English Setters, radial carpal bone fractures 321

Enrofloxacin 382, 383

Enterobacter spp. 382

Epidermal growth factor (EGF) 121

Epidurals 60

Epiphysis 12

development $14,15,16$

fractures 9

Escherichia coli 107, 375, 378, 382

ESF see external skeletal fixation

External bridging callus 34, 35

External coaptation of fractures 142-8 avoiding fracture disease $366-7$

care of 63

distal limb fractures 342

history of 1-2

maxillofacial fractures (muzzle) 151, 152

risk of fracture disease 364

External skeletal fixation (ESF) 24-7, 82-7, 99-100

ability to resist disruptive forces 72

circular ESF (CESF) 3, 27, 86

complications 3

connecting bars $25,26,85-6$

connecting clamps $25,84-5$

description 82,83

frames $25-6$

hinged systems $85,86,367$

mandible or maxilla 163

maxillofacial fractures 154

maxillomandibular ESF 151, 152

modern materials 2-3

negative-profile threaded pins 84

pins 24-5

positive-profile threaded pins 84

postoperative management 63

stress riser effect 118

systems 2-3, 5

transfixation pins 82,84

upper limb fractures $26-7$

using MIO 129

External skeletal fixation (ESF) putty 85

Extra-articular fracture 9

Extracellular matrix, generation during fracture healing 388

Extraosseous blood supply, role in bone healing 34,55

Fabellae 353, 354

fractures and other injuries 354-6, 360-1

Fasciotomy 50

Fatigue failure of implants 369-71, 373

Fatigue fractures 10

Femur 276-83

diaphyseal fractures $278-81$

distal fractures 281-3

distal physeal fractures 281-2

external skeletal fixation (ESF) 281

femoral head (epiphyseal) fracture 276

femoral neck fracture 276, 277

IM pinning 278-9

incidence of femoral fractures 276

interlocking nail technique 279, 280

ischaemic necrosis of the femoral head (INFH) 276

minimally invasive plate osteosynthesis (MIPO) 281

physeal fractures 276

plate and pin-plate fixation $279,280,281$

proximal fractures $276-7$

slipped femoral capital epiphysis (SFCE) 276, 277

supracondylar and condylar fractures 282-3

trochanteric/subtrochanteric fracture 277

Femur Operative Techniques

articular fractures of the condyle 299-300

comminuted diaphyseal fractures, pin-plate stabilization 292-3

diaphyseal fractures, open bone plating 291-2

distal physeal fracture stabilization in immature patients 295-7

epiphyseal fracture of the femoral head 284-5

ESF of the femur 294-5 greater trochanter fractures 287-8

intertrochanteric fractures 285-7

physeal fractures 285-7

spiral/long oblique diaphyseal fractures, IM pinning 289-91

subcapital fractures 285-7

supracondylar fracture stabilization in mature patients 298-9

Fentanyl 60,171

Fibroblast growth factor (FGF) 14, 121, 123

Fibrocartilage 5, 34

Fibrosarcoma 112, 114

Fibrous osteodystrophy 115, 116

Fibula see Tibia and fibula

First aid, open fractures 106-7

Fissure fracture 8

Fixation strategy

basic mechanical principles 20

factors affecting choice of 23-4

mechanics of 23-30

Fixed angle locking systems 81

Fixin system 81, 82

Fluoroquinolone antibiotics 383

Folding fractures 8

diagnostic radiography 39

in juvenile animals 61

Forceps 68

Forces acting on bones 20-2

Fracture classification

accessory carpal bone fractures 9

central tarsal bone fractures 9-10

development of classification systems 7

electronic transmission and storage of data 7

for computer analysis 10

growth plate or epiphyseal fractures (separations) 9

metacarpal/metatarsal stress fractures 10

modification of the human AO system 10-11

open fractures 10

Prieur system 10, 11

prognostic value 7,11

purpose of 7,11

role in treatment decisions 7,11

Salter-Harris classification 9

soft tissue injury classification 10

special joint fractures $9-10$

time elapsed since injury 10

Unger system 10,11

Fracture description

anatomical location 8

articular fractures 9

direction of the fracture line 8

displacement of fragments 8

early methods 7

general descriptions 7

general location 8

nature of the fracture 8

number or nature of the fragments 8

specific criteria 7-8

specific location 8

stability after reduction 9

Fracture disease 363-7

advances in fracture management 363

aetiology 363-4

articular and periarticular changes 365

clinical signs 364

definition 363

disuse osteopenia 364

effects of limb immobilization 364-5

growth disturbances 365

loss of healthy bone 364

muscle atrophy 364-5

muscle contracture $363,364-6$

pathophysiology 364-5

prevention 366-7

quadriceps contracture 363, 364, 365-6

Fracture fixation

AO principles 55

effects on blood supply 34

forces involved 21, 22

principles 55-63

Fracture gap bridging routes $34-5$

Fracture healing 32-6

cell signalling mechanisms 388

fracture site biology 387-8 
fracture site mechanics 387

generation of extracellular matrix 388

imaging 43-4

importance of a good blood supply 388

influence of interfragmentary strain (IFS) 387

influence of stress protection 387

revascularization of fracture sites 388

role of cytokines and growth factors 388

role of mesenchymal stem cells (MSCs) 386-7 see also Bone healing

Fracture management

considerations for optimal treatment 36

in osteomyelitis $380-1$

Fracture patient assessment score (FPAS) 51-3

Fracture patterns of bone 23

choice of fixation strategy 20

Fracture reduction 91-3

decision to reduce or not 24

stability after 9

Fracture surgery

aims of 55

analgesia $59-60$

application of AO principles $56-7$

arthrodesis 60

articular fractures 60,63

articular fractures in juvenile animals 61,63

aseptic techniques $57-8$

chip fractures 60

considerations in juvenile animals $60-3$

decision making in fracture stabilization 57

draping 58,59

extent of fragment reconstruction $56-7$

Halstead's principles 55, 56

hip replacement 60

importance of the soft tissue envelope 55-6

methods of fracture reduction 57

non-reconstructable fractures 60

open fractures 60

perioperative antibiotics 59

perioperative pain management $59-60$

postoperative management and rehabilitation 63

postoperative pain management 60

predisposing factors to infections 57

Salter-Harris (growth plate) fractures 62-3

total joint replacement 60

see also Basic surgical techniques and specific fractures

Fracture-associated neoplasia 47, 118 see also Neoplasia

Functional loss after fracture healing 32

Functional malunion of fractures 386

Fungal osteomyelitis 113, 378, 382, 384-5

Fusobacterium spp. 378

\section{Gabapentin 171}

Gap healing of fractures 32,33

Gas-forming organisms 376, 378

Gastrin 19

Gastrocnemius muscle injuries 354-6

Gelpi retractors 66

Gentamicin 107, 382-3 coating of implants 383

German Shepherd Dogs

congenital hyperparathyroidism 115

predisposition to infections 378,385

Glasgow Composite Pain Score 60

Glass ionomer cements, use in bone grafts 124

Glassy teeth 117

Glucocorticoids, influence on bone 17, 18

Gouges 68, 69

Granulation tissue 23, 32, 33, 34

Greenstick fractures $8,39,40,116$

Greyhounds

avulsion of the tibial tubercle 301

accessory carpal bone fractures 323-4

classification of common joint fractures $9-10$

distal limb fractures in racing dogs $342,343,345-6$

palmar and plantar sesamoid bone fractures $346-7$

radial carpal bone fractures 320

Growth disturbances, caused by immobilization 365

Growth factors 121

role in fracture healing 388

use in bone grafts $122-3$
Growth hormone (GH) 17

Growth plate

average time of closure 61

distal ulna 62

fractures $9,12,40,61,62-3$

influence of sex hormones on closure 17

Haemangiosarcoma 112, 114

Haematoma formation 33, 34

role in bone healing 56

Halsted's principles of surgery 55,56

Hard callus tissue 23, 34

Haversian canals 16, 17, 33

Haversian remodelling of bone 16, 33

Head trauma $149-65$

Hereditary neonatal primary hyperparathyroidism 115

Heterotopic skeleton 12

High-energy fractures 23

Hip replacement 60

Histoplasma capsulatum 384

History of fracture treatment 1-6

AO principles 5,6

biological approach 5-6

changes in philosophy $5-6$

developments in medicine 1,2

ESF systems 2-3, 5

internal fixation techniques 2, 3-6

Horizontal beam radiography 42

Hormonal influences on bone 17-18

Howship's lacuna 16

Humeral intracondylar fissure (HIF) 202-3

Humerus 198-226

condylar fractures 203-6

diaphyseal fractures 199-200

dicondylar fractures 205-6

distal diaphyseal and supracondylar fractures 201

distal fractures 200-6

humeral intracondylar fissure (HIF) 202-3

incidence of humeral fractures 198

incomplete ossification of the humeral condyle (IOHC) 201-3

proximal fractures 198

Salter-Harris type I and II fractures 198

Salter-Harris type III fractures 203

unicondylar fractures 203-5

Humerus Operative Techniques

diaphyseal bone plate application, medial approach 209

diaphyseal bone plate application, lateral approach 210-11

distal bone plate application, lateral approach $\mathbf{2 1 2}$

distal bone plate application, medial approach 209-10

intracondylar Y-T fractures 216-18

IM pinning 219-21

lateral condylar fractures $213-15$

linear external skeletal fixation 222-4

medial condylar fractures $\mathbf{2 1 5 - 1 6}$

medial to lateral transcondylar cortical bone screw for intracondylar fissure 226

pin-plate application 221-2

Salter-Harris type I and II proximal fractures 207

Salter-Harris type III proximal fractures 208

Hybrid ESF frames 3

Hydroxyapatite 13

alloplastic bone grafts 123

crystals 16

mineral component of bone 20

Hyoid apparatus 12

Hyperadrenocorticism 18, 45

Hyperparathyroidism 39, 61

pathological fractures $115-17$

Hypersensitivity reactions to orthopaedic implants 73

Hypertrophic non-union of fractures 45, 389-90, 392-3

IM fixation devices $3-4,75-6$

IM pins 24, 75, 93-4

ability to resist disruptive forces 72

principles of fixation 75

pin-plate technique 29

Imaging of fractures $37-47$

age of the fracture 39

apparent shortening of the bone 40

articular involvement 40, 41

assessment of healing progression 43-4 
bone density 44

bone tumour 39

carpus joint fractures 41

complete or incomplete fracture 39,40

complications of fracture repair 44-7

computed tomography (CT) 37, 41, 42

direction of fracture lines 40

evidence of avulsion 40,41

evidence of compression 40

folding fractures 39

fracture identification 38

fracture obscured by implants 44

implant positioning assessment 43

implant stability assessment 44

long bone fractures 40

magnetic resonance imaging (MRI) 37, 42

nuclear scintigraphy 38

number of bone fragments present 39

open fracture diagnosis 39,40

overriding of the fracture ends 40

pathological features 39

pelvic fractures 42

postoperative assessment 43

postoperative follow-up radiography 63

preoperative assessment $50-1$

radiographic artefacts 38,44

radiographic description $39-42$

radiography $1,2,37,38$

skull fractures 42

soft tissue changes 40

spinal fractures 42

tarsus joint fractures 41

techniques 7,10

ultrasonography 37-8

vertebral fractures 42

mpacted fracture 8

Implant-associated neoplasia 118

mplant design, and risk of osteomyelitis 383

Implant failure 45-6, 368-73

acute overload failure $368-9$

area moment of inertia (AMI) of the implant $370-1$

corrosion degradation 372-3

corrosion fatigue 373

crevice corrosion 372,373

fatigue failure 369-71, 373

fretting corrosion $372-3$

galvanic corrosion 372

mechanical failure due to metallurgic imperfections or manufacturing faults 368

mechanisms of material failure 368-73

pitting corrosion 372

screw head shearing off 368-9

selection and application of implants 368

Implants 72-86

ability to resist disruptive forces 72

adverse effects related to 73

for use in juvenile patients 61,62

hypersensitivity reactions 73

infections 73

loosening and movement 46

mechanics of 21,22

postoperative management 63

principles of fracture fixation 72

properties of implant materials $72-3$

removal 33

stainless steel implants $65,72-3$

stiffness compared to bone 21

titanium implants 73

use of fewer implants 35-6

Incomplete fracture 8

Incomplete ossification of the humeral condyle (IOHC) 201-3

Indirect bone healing 32, 33-5

angiogenesis 33-4

inflammatory phase 33-4

remodelling phase $34-5$

reparative phase 34

Induced axial micromotion 35

Infection

and non-union of fractures 391, 392-3, 394

in open fractures 107, 109, 227

predisposing factors related to surgery 57

see also Osteomyelitis
Inflammatory phase of indirect bone healing 33-4

nsulin-like growth factor 17, 123

Interarcade wiring 151

Intercanine acrylic bonding 152, 161, 162

Intercellular molecular signalling for osteoclast differentiation 16

Intercellular signalling within bone 13

Interdental acrylic or composite 161-2

Interdental acrylic splinting 152-3

interdental wiring 152-3, 161, 162

Interfragmentary strain (IFS) in fractures 32

influence on bone healing 387

Interlocking nails 24, 95

ability to resist disruptive forces 72

types and uses 75-6

Internal fixation systems

development of $2,3-6$

intramedullary devices 3-4

new approaches 5-6

orthopaedic wire 3

plates and screws $4-5$

Intracranial haemorrhage, imaging 42

Intramembranous ossification 13, 14, 33, 34

Ischaemic necrosis of the femoral head (INFH) 276

ISO (International Organization for Standardization)

classification of stainless steel types 65

Isotropic materials 20

Itraconazole 385

Jacobs chucks 70

Jamshidi bone marrow biopsy needle 114

Jaw fractures see Maxillofacial fractures, Maxillofacial Operative Techniques

Joint fractures see Articular fractures

Joint hyperlaxity 117

Joint immobilization

avoiding fracture disease $366-7$

effects of 365

Joint replacement 393, 396

Juvenile animals

age and bone development 14, 15, 16

articular fractures 61,63

avulsion fractures 62

considerations in fracture management $60-3$

fractures involving growth plates 61

malunion of fractures 60-1

Salter-Harris (growth plate) fractures 62-3

timing of closure of growth plates 61

traction epiphyseal fractures 62

K-wires 29, 30, 74

Ketoconazole 384, 385

Kirschner-Ehmer (KE) clamp 2, 84

Klebsiella spp. 107, 378

Labrador Retrievers, radial carpal bone fractures 321

Lag screws 28, 77, 101

amellar bone formation 13, 33

Lane bone plates 4

Leptin, influence on bone 18, 19

Limb development 13, 14, 15, 16

Limited contact dynamic compression plates (LC-DCP) 5, 27-9, 79

Lincosamide antibiotics 382

Linear ESF 24

mx-1 transcription factor 14

Load-deformation diagrams 20, 72

Loading, optimal level for fracture healing 35

Locking compression plates (LCP) 29, 81

Locking plate systems $5,29,80-2$

Long bones 12

development $13,14,15,16$

forces acting on 22

radiographic description of fractures 40

Longitudinal fracture 8

Mach line effect 38,44

Magnetic resonance imaging (MRI) 37, 42

Malunion of fractures $32,45,55,386,394-6$

in juvenile animals $60-1$

surgical planning 395-6 
Mandible see Maxillofacial fractures, Maxillofacial Operative Techniques

Marbofloxacin 383

Maxillofacial fractures

bi-gnathic encircling and retaining device (BEARD) 152, 153 bone plating 154, 155

challenges posed by 149

closed or minimally invasive management $151-3$

complications of jaw fracture management 159

emergency treatment 149

ESF 154

external coaptation (muzzle) 151, 152

imaging 149

interarcade wiring 151

intercanine acrylic bonding 152

interdental acrylic splinting 152-3

interdental wiring 152-3

interfragmentary wire fixation 154

labial reverse suture through buttons 152,153

management of specific fractures 155-65

management techniques 151-5

mandibular condylectomy and meniscectomy 155

mandibular fractures 155-7, 158

maxillary fractures $157-8$

maxillomandibular ESF 151, 152

open/surgical management 153-5

partial mandibulectomy/maxillectomy 155

postoperative management of jaw fractures 158

principles of treatment $149-51$

problems encountered in managing jaw fractures 158-9 salvage procedures 155

Maxillofacial Operative Techniques

application of ESF to the mandible or maxilla 163

intraoral techniques 161-2

mandibular symphyseal wiring 162-3

surgical approach to the TMJ and condylectomy 165

ventral approach to the mandibular body and plate application 164

Mechanical functions of bone 12

Mechanical manipulation of fracture healing 35

Mechanics of fixation systems 23-30

Mechanobiology of bone 16-17

Medetomidine 60

Meloxicam 60

Mesenchymal cells, osteogenesis 13-14

Mesenchymal osteoblasts 13

Mesenchymal stem cells (MSCs), role in fracture healing 34,121 , $123,386-7$

Metabolic bone disease 19

Metacarpal bones see Distal limb

Metalloproteinases 16

Metaphysis 12

fractures 8

Metatarsal bones see Distal limb

Methadone 60, 171

Methicillin-resistant Staphylococcus pseudintermedius (MRSP) 378,382

Methocarbamol 171

Methylprednisolone sodium succinate 171

Metronidazole 382

Michel's trephine 114

Micromotion

in circular ESF 27

influence on fracture healing $23,24,35$

Minimally invasive osteosynthesis (MIO) 6, 34, 56-7, 126-141 advantages over open techniques 127

basic mechanobiology 126-7

biological osteosynthesis principles 126

case selection 127-8

definitions 126-7

fracture reduction techniques $132-4$

indications $127-8$

limitations 127-8

percutaneous external fixation 126

percutaneous pinning 126, 130-1

percutaneous screw fixation $130-1$ preoperative considerations $127-8$ principles of specific fixation methods using MIO 129

Minimally invasive plate osteosynthesis (MIPO) 29, 127, 129-30 application of a plate to the femur 137-9 application of a plate to the humerus 134-5

application of a plate to the radius $136-7$ application of a plate to the tibia $140-1$

choice of implant 128, 129-30

fracture evaluation 129,130

patient selection 129

Monteggia fractures $7,228-9,230,242-3$

Morphine 60

Morphine-lidocaine-ketamine intravenous infusions 60

Multidrug-resistant organisms 378, 382

plasmid-mediated transmission of resistance traits 375

Muscle atrophy, caused by immobilization $364-5$

Muscle contracture, caused by immobilization 363, 364-6

Mycobacterium spp. 374

Myelitis 374

Myelography 42

Necrotic non-union of fractures 389,391

Negative-profile threaded pins 25, 84

Neoplasia 112-15

amputation 114

associated with fractures 47

bone biopsy 114, 115

bone tumours 39,47

clinical approach 113-15

clinical staging 113-14

diagnostic work-up 113, 114

differential diagnosis $113,386-7$

fracture associated 118

implant associated 118

incidence in dogs and cats 112

limb-sparing surgery 114-15

metastases 113-14

prognosis 114-15

radiographic assessment 113,114

treatment 114-15

Nerve blocks 60

Neurological examination, preoperative assessment 50

Neuropeptide $Y 18$

Neutering, influence on bone 17, 18

Neutralization plates 28, 103

Nocardia spp. 378

Non-functional malunion of fractures 386

Non-locking plates 27-9

Non-reconstructable fractures 60

approach to fracture surgery $56-7$

Non-self-tapping screws 77

Non-steroidal anti-inflammatory drugs (NSAIDs) 367

effects on bone healing 60

pain management 60,171

Non-surgical management of fractures $142-8$

casting 142-8

financial considerations 148

prognosis 148

splinted bandages 147

support bandages 147

without external support 147

Non-union of fractures $44-5,386,388-93$

articular non-unions 393

diagnosis $388-91$

infection 391, 392-3, 394

non-surgical treatment 393

non-viable non-unions $45,386,388,389,390-1,393$

radiographic features $388-91$

treatment $391-3$

viable non-unions $45,386,388-90,392-3$

Non-viable non-union of fractures $45,386,388,389,390-1,393$

Nutritional secondary hyperparathyroidism $61,116,117$

Obesity, effects on bone 18

Oblique fractures $8,23,32$

Oestrogen, influence on bone 17

Oligotrophic non-union of fractures 45, 389-90, 392, 393

Open but do not touch (OBDNT) approach 6, 29, 56, 109, 126

Open fractures $7,10,23,106-11$

amputation 110

arthrodesis 110,111

bone grafting 109

definition 106

diagnostic radiography 39,40

first aid 106-7

fracture management $108-10$ 
grading scheme 106

infection 107, 109, 227

management $60,106-10$

open but do not touch (OBDNT) approach 109

open wound management 107-8

prognosis 111

shearing injuries 110-11

use of antibiotics 107

wound management 107-8

Operative Techniques

application of a modified type 2 external skeletal fixator to metacarpal/metatarsal fractures $\mathbf{3 5 0}$

application of bridging plate 104

application of cerclage wire 96-7

application of dynamic compression plate 102

application of external skeletal fixation to the mandible or maxilla 163

application of lag screw 101

application of neutralization plate 103

application of tension-band wiring 98

articular fractures of the femoral condyle 299-300

atlantoaxial subluxation (C1-C2) 176-7

avulsion of the fabella $360-1$

avulsion of the popliteus 362

avulsion of the tibial tubercle 305-7

caudal thoracic fractures and luxations (T8-T12); lumbar fractures and luxations (L3-L7) 183-4

cervical fractures and luxations (C2-T1) 178-9

distal digital amputation (distal digital ostectomy) 352

distal femoral physeal fracture stabilization in immature patients 295-7

distal radial fractures - mature dog 252-3

distal radial fractures - Salter-Harris type I 253-4

dorsal approach to the carpus $\mathbf{3 3 1}$

epiphyseal fractures of the femoral head 284-5

ESF of the femur 294-5

fracture of the patella $358-9$

racture reduction 91-3

fractures of the greater trochanter 287-8

fractures of the medial and lateral malleoli 317-18

fractures of the proximal radius 244-5

fractures of the scapula body 192-4

fractures of the scapula neck 194-6

fractures of the supraglenoid tuberosity 197

fractures of the ulna with concurrent luxation of the radial head (Monteggia fractures) 242-3

harvesting of an autogenous cancellous bone graft 125

ilial body bone plating - dorsal plate application in feline patients 271

ilial body bone plating - lateral plate application 267-9

ilial body bone plating - ventral plate application in canine patients 270

IM pinning 219-21

IM pinning of metacarpal/metatarsal fractures 348-9

IM pinning of spiral/long oblique diaphyseal fractures 289-91

insertion of IM pins 93-4

insertion of interlocking nail 95

insertion of positional screw 100

internal fixation of articular fractures of the talus 337-8

internal fixation of central tarsal bone fractures 339-41

intraoral techniques 161-2

intracondylar Y-T fractures 216-18

lateral approach to the distal humerus for bone plate application $\mathbf{2 1 2}$

lateral approach to the humeral diaphysis for bone plate application 210-11

lateral condylar fractures 213-15

linear external fixation of the humerus 222-3

mandibular symphyseal wiring 162-3

medial approach to the distal humerus for bone plate application 209-10

medial approach to the humeral diaphysis for bone plate application 209

medial condylar fractures 215-16

minimally invasive application of a plate to the femur 137-9

minimally invasive application of a plate to the humerus 134-5

minimally invasive application of a plate to the radius 136-7 minimally invasive application of a plate to the tibia 140-1 open bone plating of the diaphysis 291-2

palmaromedial approach to the radial carpal bone 332

permanent nail removal (ungal crest ostectomy) 351 pin and tension-band fixation of calcaneal fractures 334-6 pin-plate application 221-2

pin-plate stabilization 105

pin-plate stabilization of comminuted diaphyseal fractures

292-3

physeal, subcapital and intertrochanteric fractures 285-7 placement of a medial to lateral transcondylar positional

cortical bone screw for humeral intracondylar fissure 226

placement of external skeletal fixators 99-100

plate application to the caudal ulna for simple fractures 239

radial diaphyseal fractures - bone plating 249-51

radial diaphyseal fractures - external fixation 247-9

sacral fractures and caudal vertebral fractures and luxations

186-7

sacroiliac luxation - sacroiliac lag screw placement 262-6

sacroiliac luxation - transilial pinning 266-7

Salter-Harris type I and II fractures of the proximal humerus 207

Salter-Harris type III fractures of the proximal humerus 208

screw fixation of type I and II accessory carpal bone fractures 333-4

separation of the distal tibial physis $315-16$

separation of the proximal tibial physis $307-8$

seventh lumbar vertebra fractures (L7) 184-6

simple acetabular fractures - bone plate stabilization 272-4

simple acetabular fractures - composite repair using

screws, wire and polymethylmethacrylate $\mathbf{2 7 5}$

stabilization of comminuted proximal ulna fractures 240-1

styloid fractures 255-6

supracondylar fracture stabilization in mature patients 298-9

supracondylar humeral fractures 224-5

surgical approach to the temporomandibular joint and condylectomy 165

reduction techniques for minimally invasive fracture surgery

132-4

tension-band application to the proximal ulna 235-8

thoracic fractures and luxations 180-1

thoracolumbar fractures and luxations (T12-L3) 181-2

tibia - ESF 313-15

tibia - IM pinning 311-13

tibia - medial bone plating 308-11

ulna diaphyseal fractures - IM pinning 245-6

ventral approach and plate application to the mandibular body 164

Ophthalmic examination, preoperative assessment 50

Opioid drugs 60, 171

Orthopaedic examination, preoperative assessment 50

Orthopaedic implants see Implants

Orthopaedic instrumentation 65-70

construction materials 65

grades of stainless steel 65

history of 65

instrument care 70

instrument manufacture 65

non-powered cutting instruments $68-9$

pin and wire placement 70

plate and screw placement $69-70$

power tools 66-8

sterilization 57-8

types and uses 65-71

wire twisters 70

Orthopaedic wire $72,73-5$

area moment of inertia (AMI) 74

cerclage wiring $72,73-4$

fixation 3, 29-30

instruments to use with 70

K-wires 74

Rush pins 74-5

tension-band wiring 72,74

wires used for pinning 74-5

Osteitis 374

Osteoarthritis 365

in articular fractures 55, 60

secondary 32

Osteoblasts $13,14,16-17,18,33,34,123$

Osteocalcin 13

Osteoclasts 16, 17, 18-19, 33, 34, 123

Osteoconduction 120

Osteocytes 13, 14

Osteogenesis 117, 120

Osteogenic mesenchymal cells 13-14 
Osteoid 13, 16, 33

Osteoinduction 120

Osteointegration 120

Osteomyelitis 46-7, 374-85

acute and chronic forms 374

antibiotic coating of implants 383

antibiotic therapy $379,382-3,384-5$

bacterial inoculation routes $374-5$

bacteriology 378

biofilm development 374-5

classification 374

clinical and laboratory findings 376

definition 374

diagnosis $376-8$

discospondylitis $384-5$

effect of implant design 383

factors affecting development of 383

fungal osteomyelitis $378,382,384-5$

gas-forming organisms 376,378

haematogenous osteomyelitis $374,383-5$

histopathology 378

imaging 376-8

pathogenesis of post-traumatic osteomyelitis 374-5

patient history 376

perioperative antibiotics 383

post-traumatic osteomyelitis $107,374-5$ treatment 378-83

risk factors 374,375

sources of infection 376

treatment of post-traumatic osteomyelitis $378-83$

vertebral osteomyelitis $384-5$

Osteons 16, 21, 33

Osteopenia

caused by stress protection 61

related to fixation 36

related to immobilization $44,46,364$

related to parathyroid hormone 115

Osteoporotic bone fractures 29

Osteoprogenitor cells 33-4

Osteopromotion 120

Osteoprotegerin (OPG) 16

Osteosarcoma 39

associated with fracture or implant $47,73,118$

pathological fractures 112-15

Osteostixis 393

Osteotomes 68-9

Oxterix transcription factor 13

Pain management

analgesia for fractures $59-60$

perioperative 60

postoperative 60

sensitization (wind-up) 171

spinal injuries 171

types of analgesic 171

Pain scoring systems 60

Paracetamol 60

Parathyroid gland hyperplasia 115-16

Parathyroid hormone (PTH) 18-19 effects on bone 115-16

Partial articular fracture 9

Partial mandibulectomy/maxillectomy 155

Partially threaded connecting bars 85

Pasteurella spp. 378

Patella 353

Patellar fractures 353-4, 358-9

Pathological fractures $7,8,112-18$ bone cysts 117-18

fracture-associated sarcoma 118

hyperparathyroidism 115-17

in juvenile animals 61

neoplasia 112-15

osteogenesis imperfecta 117

patient history 112

prodromal lameness 112

radiographic features 39

stress riser effect 118

underlying aetiologies 112

Patient assessment see Preoperative assessment

Pelvic fractures $147,257-75$

acetabular fractures 260-1 aetiology 257

assessment for surgical or non-surgical management 257-8

emergency care and stabilization 257

ilial body fractures 260

imaging 42, 227

incidence 257

ischium fractures 261

non-surgical management 258

postoperative management 261

pubis fractures 261

sacroiliac luxation 258-60

surgical management 258-61

Pelvic Operative Techniques

ilial body, dorsal bone plate application in feline patients 271

ilial body, lateral bone plate application 267-9

ilial body, ventral bone plate application in canine patients

sacroiliac luxation, sacroiliac lag screw placement 262-6

sacroiliac luxation, transilial pinning 266-7

simple acetabular fractures, bone plate stabilization 272-4

simple acetabular fractures, composite repair using screws, wire and polymethylmethacrylate $\mathbf{2 7 5}$

Peptostreptococcus spp. 378

Percutaneous external fixation 126

Percutaneous pinning 126, 130-1

Percutaneous screw fixation 130-1

Periosteal elevators 66

Periosteum, ossification of elevated/stripped areas 47

Periostitis 374

Pethidine 60

Phalanges see Distal limb

Phenoxybenzamine 174

Physeal cartilage 12

Physeal fractures see Growth plate fractures

Physes see Growth plates

Physiotherapy 63, 367

Piezoelectric fields within bone 34, 393

role in cellular signalling 17

Pin and wire placement, instruments for 70

Pin-bone interface, stresses on 24-5

Pin-cerclage wire fixation 29-30

Pin cutters 70

Pin-plate combination fixation 6, 29, 105

Pinning, wires used for 74-5

Pins, types for ESF 24-5

Pituitary dwarfism 18

Plate and screw fixation 27-9

instruments for 69-70

plate length 27

plates and screws 4-5

staged selective screw removal 35-6

see also Bone plates, Minimally invasive plate osteosynthesis (MIPO)

Plate-rod fixation 29, 75

Plate screws 77

Platelet-derived growth factor (PDGF) 33, 121, 123

Platelet-rich plasma (PRP) 123

Podosomes 16

Pointers, radial carpal bone fractures 321

Polar moment of inertia (PMI) 21, 22

Polyaxial locking plates 81,82

Polyostotic disease 378

Popliteal sesamoid bone

location and displacement 353, 356, 362

Positional screws 77, 100

Positive-profile threaded pins 25, 84

Postoperative management of fracture patients 63

Pott's fracture 7

Power tools for orthopaedic surgery 66-8

drills 66-7, 68

modular power tools 67

saws 67-8

Preoperative assessment 49-54

abdominal examination 49

detailed examination of body systems 49-53

forming a treatment plan 53-4

fracture patient assessment score (FPAS) 51-3

imaging the fracture region 50-1

initial patient examination 49

neurological examination 50

ophthalmic examination 50

orthopaedic examination 50 
telephone advice 49

thoracic examination 49

vascular structural integrity 50

Primary bone union see Direct healing of fractures

Primary hyperparathyroidism 19, 115

Primary loss of reduction 27

Prodromal lameness 112

Proteus spp. 107, 378, 382

Proximal fracture 8

Pseudoarthrosis 390, 392

Pseudomonas spp. 107, 375, 378, 382, 383

Quadriceps contracture 363, 364, 365-6

Radiographic description of fractures 39-42

Radiographic union 386

Radiography 37

artefacts 38,44

development of 1,2

diagnosis of fractures 38

postoperative follow-up 63

principles of radiography of fractures 37 see also Imaging of fractures

Radius and ulna 227-34

anatomy 227

diaphyseal fractures 230-4

distal fractures 234

distal ulnar growth plate 62

evaluation of the patient 227

incidence of fractures 227

Monteggia fractures 228-9, 230

particular features in cats 227

proximal fractures 227-30

radial head fractures 229

Salter-Harris type I fractures 230

styloid fractures 234

Radius and ulna Operative Techniques

distal radial fractures, mature dog 252-3

distal radial fractures, Salter-Harris type I 253-4

Monteggia fractures 242-3

plate application to the caudal ulna for simple fractures $\mathbf{2 3 9}$

proximal radial fractures 244-5

radial diaphyseal fractures, external fixation 247-9

radial diaphyseal fractures, bone plating 249-51

stabilization of comminuted proximal ulna fractures 240-1

styloid fractures 255-6

tension-band application to the proximal ulna $235-8$

ulnar diaphyseal fractures, intramedullary pinning 245-6

ulnar fracture with concurrent luxation of the radial head

$$
\text { 242-3 }
$$

RANK ligand (RANKL) 16, 18

Reconstruction plates 79

Reduction of fractures, methods 57

Regeneration of bone 32

Remodelling phase of indirect bone healing 34-5

Renal secondary hyperparathyroidism 116

Reparative phase of indirect bone healing 34

Retractors 65-6

Revascularization of fracture sites 388

Ribs 12, 13

Robert Jones bandage 63

Rongeurs 68, 69

Rottweilers

palmar and plantar sesamoid bone fractures $346-7$

thoracic limb lameness 347

'Rubber jaw' condition 115

Runx2 (Cbfa1) transcription factor 13, 14

Rush pins $74-5$

Sacroiliac joint injuries 257

Sacroiliac joint luxation

sacroiliac lag screw placement 262-6

surgical management 258-60

transilial pinning 266-7

Salter-Harris fractures $61,62-3$ classification 9

Sarcoma, associated with fracture or implant 118 see also Osteosarcoma

Saws for orthopaedic surgery $67-8$

Scapula 188
Scapular fractures 147, 188-97

concurrent injuries 188

diagnosis 188-9

imaging 188-9

neurovascular structures in the scapular region 188

scapular body fractures 189-90

scapular neck fractures 190-1

supraglenoid tuberosity fractures 191-2

Scapular Operative Techniques

scapular body fractures 192-4

scapular neck fractures 194-6

supraglenoid tuberosity fractures 197

Schroeder-Thomas splint 2, 147

Sciatic neuropraxia 365

Screwdrivers (orthopaedic) 69-70

Screws see Bone screws

Secondary fractures 7,8 see also Pathological fractures

Secondary bone healing 23

Secondary hyperparathyroidism 115-16, 117

Secondary osteonal remodelling 33

Self-tapping screws 77

Septic arthritis 384

Sequestrum/involucrum formation 46-7

Sesamoid bones 13, 353

fractures 353

of the distal limb 342

see also specific sesamoid bones

Sex hormones, influence on bone 17

Shear stress 20

Shearing injuries 110-11

Simple (closed) fractures 7 approach to fracture surgery 56

Skeletal anatomy 12-13

$\mathrm{SK}^{\mathrm{TM}}$ system clamp $84-5$

Skull 12

development 13

fractures 149-65

imaging 42

Slings 147

Slipped femoral capital epiphysis (SFCE) 276, 277

Soft callus tissue 23, 34

Soft tissue

atrophy 47

contracture 32

injuries 55-6

injury classification 10

radiographic imaging of changes 40

role in fracture healing $5,6,55-6$

trauma in high-energy fractures 23

Sonic hedgehog (Shh) gene 14

Spica splint 147

Spinal injuries 166-87

aetiology 166

cage confinement 173

computed tomography (CT) 169

differential diagnosis 167

emergency care 166

evaluation of the patient 166-7

external splinting 172

grading system for spinal cord injury 166-7

imaging 42, 167-169

magnetic resonance imaging (MRI) 169

management knowledge required 169-71

management of secondary spinal cord injury 171

management of spinal cord compression 172

management of vertebral instability 172

management principles 171-2

myelography 168

neurological examination $166-7$

non-surgical management 172-3

pathophysiology of acute spinal injury 169-70

physical examination 166

progressive myelomalacia 167

spinal biomechanics 170

spinal cord injury grading system 166-7

spinal pain management 171

spinal shock 167

survey radiography 168, 169

types of vertebral injury 170, 171

Spinal injury surgery 173-4

biomechanics of spinal fixation techniques 173 
choice of fixation technique $173-4$

decompressive surgery 174

postoperative management $174-5$

prognosis 175

screw/pin implantation in vertebrae 174

Spinal Operative Techniques

atlantoaxial subluxation (C1-C2) 176-7

caudal thoracic fractures and luxations (T8-T12) 183-4

caudal vertebral fractures and luxations 186-7

cervical fractures and luxations (C2-T1) 178-9

lumbar fractures and luxations (L3-L7) 183-4

sacral fractures 186-7

seventh lumbar vertebra fractures (L7) 184-6

thoracic fractures and luxations (T1-T8) 180-1

thoracolumbar fractures and luxations (T12-L3) 181-2

Spiral fractures $8,23,32$

Splinted bandages 147

Splints

avoiding fracture disease $366-7$

history of fracture splinting 1

use in juvenile animals 62

Springer Spaniels

incomplete ossification of the humeral condyle (IOHC) 201-3

radial carpal bone fractures 321

Stader splint 2

Staging down of an external fixator 35

Stainless steel ESF connecting bars 85

Stainless steel implants $65,72-3$

Staphylococcus spp. 107, 374, 375, 378, 382

Steinmann pins $4,74,75$

Sterilization of surgical equipment $57-8$

Sternum 12

development of sternebrae 13

Stiffness, definition 20, 21

Strain

definition 21

optimal level for fracture healing 35

Strain rate, effect on bone 23

Strain theory of interfragmentary bone healing 23,32

Streptococcus spp. 378

Stress, definition 20

Stress fractures 10,38

Stress protection, effects on bone healing 46, 61, 387

Stress riser effect 118

and pin failure 25

Stress-strain diagrams 21,72

String-of-pearls (SOP) plates 29, 81

Subcapital fracture 8

Subchondral bone cysts $117-18$

Subtrochanteric fracture 8

Support bandages 147

Supracondylar fractures 224-5

Supracondylar plates 80

Surgery see Basic surgical techniques, Fracture surgery and specific fractures

Surgical instruments see Orthopaedic instrumentation

T-fracture 8

T-plates 79

Tantalum 124

Tarsal arthrodesis plates 80

Tarsal bones, anatomy of the tarsus 324

Tarsal fractures

anatomy of the tarsus 324

calcaneus $324-7$

central tarsal bone 9-10, 329-30

diagnosis 319,324

imaging 41,324

incidence of 319

plantar process of the central tarsal bone 330

preoperative planning 319

second, third and fourth tarsal bone fractures 330

shearing injuries of the tarsus 330

talus 327,328

Tarsal Operative Techniques

articular fractures of the talus, internal fixation $\mathbf{3 3 7 - 8}$

calcaneal fractures, pin and tension-band fixation 334-6

central tarsal bone fractures, internal fixation 339-41

Temporomandibular joint (TMJ) injuries 149

see also Maxillofacial fractures

Tension-band wiring 29, 30, 72, 74, 98
Terrier breeds

abnormal location of the medial fabella 355

avulsion of the tibial tubercle 301

Testosterone, influence on bone 17

Thoracic examination, preoperative assessment 49

Thyroid C-cells 19

Thyroid hormone, influence on bone 17

Tibia and fibula 301-18

avulsion of the tibial tubercle 301

diaphyseal fractures $302-3$

distal fractures 303-4

distal physeal separation 303

fracture incidence and causes 301

fracture of the proximal fibula 302

fracture of the proximal tibial metaphysis-diaphysis 302

lateral or medial malleolar fractures 303-4

proximal fractures $301-2$

proximal tibial physeal separation 301-2

Tibia and fibula Operative Techniques

avulsion of the tibial tubercle $305-7$

medial and lateral malleolar fractures 317-18

separation of the distal tibial physis $315-16$

separation of the proximal tibial physis 307-8

tibia, ESF 313-15

tibia, IM pinning 311-13

tibia, medial bone plating 308-11

tibial plateau levelling osteotomy (TPLO) 73

Ticarcillin 382

Tissue engineering, for bone grafting 123

Titanium ESF connecting bars 85

Titanium implants 73

Torsional forces on bones and implants 21, 22

Total joint replacement 60

Traction epiphyseal fractures in juvenile animals 62

Tramadol 60

Transdermal fentanyl patches 60

Transfixation pins 82,84

Transforming growth factor beta (TGF- $\beta$ ) 13, 33, 34, 121, 122, 123

Transverse fractures $8,23,32$

Traumatic myocarditis 49

Triage 106-7

Tricalcium phosphate (TCP) bone graft material 123

Tumours see Neoplasia

Type X collagen 14

Uberschwinger effect 44

Ulna see Radius and ulna

Ultrasonography 37-8, 393

Unstable fractures, healing process $33-5$

Vancomycin coating of implants 383

Vascular endothelial growth factor (VEGF) 33, 121, 123

Vascular supply of bones 55

Vascularity and fracture healing 32

Ventral approach to the mandibular body and plate application

$$
164
$$

Vertebrae, development of 13

Vertebral column 12

Vertebral fractures, imaging 42

Vertebral osteomyelitis 384-5

Veterinary T-plates 79

Veterinary cuttable plates (VCP) 79

Viable non-union of fractures $45,386,388-90,392-3$

Viscoelastic behaviour of cortical bone 23

Vitamin D 18, 19, 116

Wedge biopsy 114

Wedge fragment fracture 8

Wire see Orthopaedic wire

Wnt7a gene 14

Wolff's law 16, 34

Woven bone 13, 23, 34

X-rays, discovery of 1

Y-fracture 8

Young's modulus of elasticity 21 